\title{
Maternal vitamin D deficiency and the risk of autism spectrum disorders: population-based study
}

Cecilia Magnusson, Michael Lundberg, Brian K. Lee, Dheeraj Rai, Håkan Karlsson, Renee Gardner, Kyriaki Kosidou, Stefan Arver and Christina Dalman

\section{Background}

Maternal vitamin D deficiency may increase risk of autism spectrum disorder (ASD), but direct evidence is lacking.

\section{Aims \\ To clarify the relationship between maternal vitamin $D$ deficiency and offspring risk of ASD with and without intellectual disability.}

\section{Method}

Using a register-based total population study ( $N=509$ 639), we calculated adjusted odds ratios (aORs) and 95\% confidence intervals (CIs) of ASD with and without intellectual disability in relation to lifetime diagnoses of maternal vitamin D deficiency Although rare, such deficiency was associated with offspring risk of ASD with, but not without, intellectual disability (aORS
2.51,95\% Cl 1.22-5.16 and 1.28, 0.68-2.42). Relationships were stronger in non-immigrant children.

\section{Conclusions}

If reflecting associations for prenatal hypovitaminosis, these findings imply gestational vitamin D substitution as a means of ASD prevention.

\section{Declaration of interest}

None.

\section{Copyright and usage}

(c) The Royal College of Psychiatrists 2016. This is an open access article distributed under the terms of the Creative Commons Non-Commercial, No Derivatives (CC BY-NC-ND) licence.
Autism spectrum disorders (ASDs) are developmental disorders associated with a high individual and societal burden, but their aetiology is poorly understood. Environmental and genetic factors appear equally important, ${ }^{1}$ although ASD with and without intellectual disability may in part have different origins. ${ }^{2,3}$

In high-latitude countries such as Sweden, children of mothers with dark complexion are at particularly elevated risk of ASD with intellectual disability. ${ }^{3}$ Because such women often have very low vitamin D levels due to melanin absorption of UVB radiation, maternal vitamin $\mathrm{D}$ deficiency has been suggested to contribute to ASD risk in offspring. ${ }^{4,5}$ Indeed, experimentally induced vitamin D deficiency in pregnant dams is reported to affect foetal neurodevelopment and behaviour in adult offspring in rats, ${ }^{4}$ and a recent study demonstrated lower neonatal vitamin $\mathrm{D}$ levels in children with ASD compared with their siblings. ${ }^{6}$

Early life vitamin D deficiency is thus a possible risk factor for ASD, but direct support of an association is lacking. ${ }^{4}$ Maternal hypovitaminosis D is treatable, thus investigation of the matter is warranted. We report here on the relationship between secondary care diagnosis of lifetime maternal vitamin D deficiency and offspring risk of ASD with and without intellectual disability, while addressing the influence of ethnicity. We use data from the Stockholm Youth Cohort (SYC), which allow for detailed case ascertainment in the total population of young people in Stockholm, Sweden.

\section{Method}

We conducted a cohort study using the SYC, comprising all Swedish-born individuals aged 4-17 years who were resident for at least 4 years in Stockholm County between 2001 and 2011 and had complete covariate data $(N=509639){ }^{7}$ Prospectively recorded data on probands and their relatives were retrieved from registers as previously described. ${ }^{7}$
We identified cases from several national and regional registers, using a validated approach covering all known pathways of ASD diagnosis and care in Stockholm County. ${ }^{7}$ The National Patient Register contains the dates and discharge diagnoses of all in-patient (since 1973) and specialist out-patient care (since 2001) in Sweden. ${ }^{7}$ Using this source, we defined exposure as a lifetime-recorded diagnosis of unspecified vitamin D deficiency (ICD-10 E55.9 or ICD-9 268.9), corresponding to a serum 25-hydroxyvitamin D level of less than $25 \mathrm{nmol} / \mathrm{L}$ (with clinical manifestations such as osteomalacia, muscle aches and weakness), in the biological mother.

We considered other parental characteristics described below (also see Table 1) as potential confounders or mediators based on their associations with ASD and vitamin D deficiency. ${ }^{5,8,9}$

\section{Statistical analysis}

All analyses were conducted using SAS 9.4. We first calculated frequencies and means of case status and other characteristics according to lifetime maternal vitamin D deficiency, with Pearson's chi-square tests to compute the difference between proportions and $t$ tests to test the difference between means. Using logistic regression models, we then derived odds ratios (ORs) and their 95\% confidence intervals (CIs) for all ASDs, and dichotomised into ASD with or without intellectual disability, in relation to the exposure. Cluster robust standard errors accounted for non-independence within family clusters. We first estimated associations adjusting only for gender and year of birth, and then after additional adjustment for parental ages, income, education, and lifetime histories of diagnosed affective disorders, non-affective psychosis, ASD, attention deficit/ hyperactivity disorder, intellectual disability, epilepsy and prescriptions for anticonvulsants. We then included maternal country of birth in a third model. Lastly, we evaluated whether lifetime maternal vitamin D deficiency was differently associated with ASD among children with and without maternal histories of migration. We calculated chi-square tests as a test for homogeneity of associations 
Table 1 Study population characteristics in relation to lifetime maternal history of diagnosed vitamin D deficiency

\begin{tabular}{|c|c|c|c|c|}
\hline \multirow[b]{2}{*}{ Characteristic } & \multicolumn{2}{|c|}{ Maternal history of vitamin D deficiency } & \multirow[b]{2}{*}{$P$} & \multirow[b]{2}{*}{ All } \\
\hline & No & Yes & & \\
\hline Individuals, $n$ (\%) & $509092(99.9)$ & $547(0.1)$ & & $509639(100.0)$ \\
\hline Cases of ASD with intellectual disability, $n(\%)$ & $2463(0.5)$ & $13(2.4)$ & $<0.0001$ & $2476(0.5)$ \\
\hline Cases of ASD without intellectual disability, $n$ (\%) & $7396(1.4)$ & $10(1.8)$ & 0.46 & $7406(1.4)$ \\
\hline Male (\%) & 51.3 & 52.6 & 0.51 & 51.3 \\
\hline Maternal age, years: mean & 29.9 & 28.5 & $<0.0001$ & 29.9 \\
\hline Paternal age, years: mean & 32.8 & 34.0 & $<0.0001$ & 32.8 \\
\hline \multicolumn{5}{|l|}{ Maternal country of birth (\%) } \\
\hline Sweden & 76.3 & 19.2 & $<0.0001$ & 76.2 \\
\hline Europe & 8.3 & 4.6 & 0.002 & 8.3 \\
\hline Outside Europe & 12.8 & 38.9 & $<0.0001$ & 12.9 \\
\hline Sub-Saharan Africa & 2.6 & 37.3 & $<0.0001$ & 2.6 \\
\hline Lowest quintile of disposable family income (\%) & 14.8 & 35.6 & $<0.0001$ & 14.8 \\
\hline Elementary maternal education (\%) & 15.6 & 43.1 & $<0.0001$ & 15.6 \\
\hline Elementary paternal education (\%) & 16.8 & 32.7 & $<0.0001$ & 16.8 \\
\hline Born small for gestational age (\%) & 2.5 & 3.7 & 0.09 & 2.5 \\
\hline Premature birth (<37 weeks, \%) & 5.9 & 6.2 & 0.07 & 5.9 \\
\hline Maternal history of neuropsychiatric disorder (\%) & 2.4 & 9.3 & $<0.0001$ & 2.4 \\
\hline Paternal history of neuropsychiatric disorder (\%) ${ }^{a}$ & 2.0 & 2.0 & 0.98 & 2.0 \\
\hline Maternal history of affective disorder $(\%)^{b}$ & 15.4 & 36.8 & $<0.0001$ & 15.4 \\
\hline Paternal history of affective disorder $(\%)^{b}$ & 8.1 & 10.8 & 0.02 & 8.1 \\
\hline Maternal history of epilepsy (\%) & 1.0 & 1.5 & 0.25 & 1.0 \\
\hline Paternal history of epilepsy (\%) & 1.0 & 0.7 & 0.52 & 1.0 \\
\hline \multicolumn{5}{|c|}{$\begin{array}{l}\text { a. Parental histories of neuropsychiatric disorder include any in-patient or secondary care out-patient record of diagnosed non-affective psychosis, bipolar disorder, attention-deficit } \\
\text { hyperactivity disorder, ASD, or intellectual disability. } \\
\text { b. Parental histories of affective disorder include any in-patient or secondary care out-patient record of diagnosed affective disorder except for bipolar disorder. } \\
\text { ASD, autism spectrum disorder. }\end{array}$} \\
\hline
\end{tabular}

with ASD, respectively, with and without intellectual disability, and between Swedish-born children versus children of migrant mothers.

\section{Results}

By the end of follow-up in 31 December 2011, 9882 ASD cases were identified including 2476 with intellectual disability and 7406 without intellectual disability. Lifetime maternal vitamin D deficiency was rare according to secondary care diagnoses and recorded in only 547 individuals $(0.1 \%)$. It was strongly associated with socioeconomic and medical parental characteristics (Table 1) including maternal psychiatric disorders, but not with preterm or small for gestational age births.

Diagnosed lifetime maternal vitamin D deficiency was associated with risk of ASD and, in particular, of ASD with intellectual disability ( $P$ for homogeneity 0.17 ) (Table 2 ), although there was some attenuation upon adjustment for parental characteristics. Associations varied with maternal migration status ( $P$ for homogeneity 0.05$)$ as risks were more pronounced in native-born children (ORs being 7.08, 95\% CI 2.96-16.94 and 2.15, 0.85-5.45 for ASD with and without intellectual disability, respectively).

\section{Discussion}

This study is, to our knowledge, the first population-based study to estimate the relationship between maternal vitamin $\mathrm{D}$ deficiency and the risk of ASD in a child. We found a positive association which was especially noticeable for ASD with intellectual disability, and for children of non-immigrant mothers.

Our findings should, however, be interpreted with caution. Although we had access to a large sample size, estimates of associations were based on low numbers. Second, maternal vitamin D status was identified from secondary care records, and therefore under-ascertained and perhaps differentially so with regard to ASD risk factors. Indeed, diagnosed maternal vitamin D deficiency cooccurred with such factors including parental psychiatric histories ${ }^{10}$ (including depression, a proxy also for in utero exposure to antidepressants, shown to, rather than the underlying disorder, increase the risk of ASD), ${ }^{11}$ immigration status ${ }^{3}$ and disadvantageous social position. ${ }^{8}$ Hence, residual confounding cannot be ruled out. Lastly, we used vitamin D deficiency recorded at any time as the exposureand not timed to pregnancy, as no cases diagnosed before the birth of a child were identified. Therefore, reverse causality may be a

Table 2 Odds ratios (ORs) and 95\% confidence intervals (CIS) for autism spectrum disorder (ASD), with and without comorbid intellectual disability, in relation to lifetime maternal history of diagnosed vitamin D deficiency

\begin{tabular}{|c|c|c|c|c|}
\hline Vitamin D deficiency & Cases & OR $(95 \% \mathrm{Cl})^{\mathrm{a}}$ & OR $(95 \% \mathrm{Cl})^{\mathrm{b}}$ & OR $(95 \% \mathrm{Cl})^{\mathrm{C}}$ \\
\hline \multicolumn{5}{|l|}{ All ASD } \\
\hline No & 9859 & 1.00 (ref) & 1.00 (ref) & 1.00 (ref) \\
\hline Yes & 23 & $2.20(1.35-3.58)$ & $1.70(1.03-2.79)$ & $1.78(1.08-2.92)$ \\
\hline \multicolumn{5}{|c|}{ ASD with intellectual disability } \\
\hline No & 2463 & 1.00 (ref) & 1.00 (ref) & 1.00 (ref) \\
\hline Yes & 13 & $5.08(2.53-10.20)$ & $3.55(1.76-7.18)$ & $2.51(1.22-5.16)$ \\
\hline \multicolumn{5}{|c|}{ ASD without intellectual disability } \\
\hline No & 7396 & 1.00 (ref) & 1.00 (ref) & 1.00 (ref) \\
\hline Yes & 10 & $1.23(0.67-2.28)$ & $0.99(0.52-1.87)$ & $1.28(0.68-2.42)$ \\
\hline \multicolumn{5}{|c|}{$\begin{array}{l}\text { a. Adjusted for year of birth and gender. } \\
\text { b. Additionally adjusted for disposable income, maternal and paternal age and level of education at birth, maternal and paternal lifetime histories of diagnosed affective disorders, non- } \\
\text { affective psychosis, ASD, attention-deficit/hyperactivity disorder, intellectual disability, epilepsy and prescriptions for anticonvulsants. } \\
\text { c. Additionally adjusted for maternal country of birth. }\end{array}$} \\
\hline
\end{tabular}


concern. No cases of vitamin D deficiency were recorded before the birth of a child. However, given that vitamin D deficiency among pregnant women in Sweden is detected only when outright screened for, ${ }^{12}$ many cases of deficiency are likely to manifest well before any official diagnosis is recorded.

Our finding of a stronger association between maternal hypovitaminosis $\mathrm{D}$ and $\mathrm{ASD}$ with intellectual disability than without intellectual disability is in line with migrant studies, demonstrating that offspring risk of severe forms of ASD - but not Asperger's syndrome or ASD without intellectual disability - is increased in migrant populations where vitamin $\mathrm{D}$ deficiency is highly prevalent. ${ }^{3-6,8}$ There is also mounting evidence that environmental factors are more relevant (than genetic factors) for the development of lower-functioning ASD. ${ }^{2}$ We, as well as others, ${ }^{6}$ further observed heterogeneity in the associations between vitamin D deficiency and ASD between children with and without maternal migrant history. This discrepancy might be due to a limited range of exposure in children of immigrant mothers, as the majority of children and pregnant mothers with migrant background have neonatal/gestational vitamin D deficiency, according to recent Swedish reports. ${ }^{6,11}$

Causality cannot be inferred from our study. There are, however, biological underpinnings of a true association as experimental studies indicate critical roles of vitamin $\mathrm{D}$ in the development and functioning of the brain. ${ }^{4}$

In summary, our results support, but do not prove, the hypothesis that maternal vitamin D status is implicated in the aetiology of ASD with comorbid intellectual disability. As vitamin D deficiency in pregnancy is both prevalent and treatable, further investigation of this association is needed.

Cecilia Magnusson, MD, PhD, Kyriaki Kosidou, MD, PhD, Christina Dalman, MD, PhD, Department of Public Health Sciences, Karolinskalnstitutet, Stockholm, Sweden; Centre for Epidemiology and Community Medicine, Stockholm County Council, Solna, Sweden; Michael Lundberg, MPH, Department of Public Health Sciences, Karolinskalnstitutet, Stockholm, Sweden; Brian K. Lee, PhD, Department of Epidemiology and Biostatistics, A.J. Drexel Autism Institute, Drexel University School of Public Health, Philadelphia, PA, USA; Dheeraj Rai, MRCPsych, PhD, Department of Public Health Sciences, Karolinskalnstitutet, Stockholm, Sweden; Centre for Academic Mental Health, School of Social and Community Medicine, University of Bristol, Bristol, UK; Avon and Wiltshire Partnership Mental Health NHS Trust, Bristol, UK; Håkan Karlsson, PhD, Department of Neuroscience, Karolinskalnstitutet, Stockholm, Sweden; Renee Gardner, PhD, Department of Public Health Sciences, Karolinskalnstitutet, Stockholm, Sweden; Stefan Arver, MD, PhD, Department of Medicine, Karolinskalnstitutet, Stockholm, Sweden

Correspondence: Cecilia Magnusson, Department of Public Health Sciences, Karolinskalnstitutet, SE-171 77, Stockholm, Sweden. Tel: +46-(0)-8-12337133. Email: Cecilia.magnusson@ki.se

First received 8 Jan 2016, final revision 8 Jan 2016, accepted 11 Mar 2016

\section{Funding}

The data linkages and staff costs for this study have been supported by grants from the Swedish Research Council (523-2010-1052), the Swedish Regional agreement on medical training and clinical research (ALF) and by a grant from the National Institutes of Health, USA (NIH 1 R21 ES023760-01A1, 'Early life vitamin D levels and risk of autism spectrum disorders')

No funder had any role in the study design; data collection, analysis, or interpretation: in the writing of the report; or in the decision to submit the article for publication. The views expressed are those of the authors and not necessarily those of any of the funders or organisations they represent.

\section{References}

1 Sandin S, Lichtenstein P, Kuja-Halkola R, Larsson H, Hultman CM, Reichenberg A. The familial risk of autism. JAMA 2014; 31: 1770-7.

2 Robinson EB, Samocha KE, Kosmicki JA, McGrath L, Neale BM, Perlis RH, et al. Autism spectrum disorder severity reflects the average contribution of de novo and familial influences. Proc Natl Acad Sci USA 2014; 111: 15161-5.

3 Magnusson C, Rai D, Goodman A, Lundberg M, Idring S, svensson A, et al. Migration and autism spectrum disorder: population-based study. Br J Psychiatry 2012; 201: 109-15.

4 Eyles DW, Burne TH, McGrath J. Vitamin D, effects on brain development, adult brain function and the links between low levels of vitamin $D$ and neuropsychiatric disease. Front Neuroendocrinol 2013; 34: 47-64.

5 Holick MF. Vitamin D deficiency. N Eng/ J Med 2007; 357: 266-81.

6 Fernell E, Bejerot S, Westerlund J, Miniscalco C, Simila H, Eyles D, et al. Autism spectrum disorder and low vitamin D at birth: a sibling control study. Mol Autism 2015; 6: 3.

7 Idring S, Rai D, Dal H, Dalman C, Sturm H, Zander E, et al. Autism spectrum disorders in the Stockholm Youth Cohort: design, prevalence and validity. PLOS One 2012; 7: e41280.

8 Rai D. Environmental risk factors for autism spectrum disorders. PhD thesis, Karolinskalnstitutet, 2013

9 Aghajafari F, Nagulesapillai T, Ronksley PE, Tough SC, O'Beirne M, Rabi DM. Association between maternal serum 25-hydroxyvitamin $\mathrm{D}$ level and pregnancy and neonatal outcomes: systematic review and meta-analysis of observational studies. BMJ 2013; 346: f1169.

10 Sullivan PF, Magnusson C, Reichenberg A, Boman M, Dalman C, Davidson M, et al. Family history of schizophrenia and bipolar disorder as risk factors for autism. Arch Gen Psychiatry 2012; 69: 1099-103.

11 Rai D, Lee BK, Dalman C, Golding J, Lewis G, Magnusson C. Parental depression, maternal antidepressant use during pregnancy, and risk of autism spectrumdisorders: population based case-control study. BMJ 2013; 346: f2059.

12 Bergström I, Palmér M, Persson J, Blanck A. Observational study of vitamin D levels and pain in pregnant immigrant women living in Sweden. Gynecol Endocrinol 2014; 30: 74-7. 